\title{
Addressing gender inequality in academia
}

\section{The role of Irish funding agencies}

\author{
Annie Doona
}

\section{Introduction}

The story of Irish higher education could be summarised as 500 years of male dominated mono-cultural stasis followed by 100 years of near glacial progress, following the admission of the first female students to Trinity College Dublin and University College Dublin in the early 1900s. A number of areas of inequality still exist, including the under-representation of women in senior academic posts. The landscape of higher education in Ireland, as elsewhere, is complex and multidimensional. Its many players include academic presidents, funders, regulatory bodies, policymakers and politicians, private equity, influential individuals and of course students, as individuals, teams and as represented by student unions. This chapter examines the role of funding agencies for Irish higher education institutions in a changing landscape and how they will implement their stated policies. Do they see themselves as advocates and agents of change or as observers in which their role is to monitor and report? If the former, then what actions can they take, what levers do they control and are they willing to use their influence and resources, including the threat of withholding funding, to challenge preconceptions of how things should be done? Irish HEA statistics show that, despite the fact that women held 52 per cent of all lecturing posts, they occupied only 18 per cent of professorial posts in Ireland in 2013, rising to only 26 per cent in 2018. Ireland has yet to appoint a female university president and only three of the 11 Institutes of Technology (IoTs) are currently led by women. Pay grades also reflect the promotions imbalance, with women accounting for just 31 per cent of those paid over $€ 106,000$ in universities and only 21 per cent of those in IoTs in 2018 (HEA 2019a).

The Irish National Strategy for Higher Education to 2030 contains no reference to gender equality, though it does reference access to learning for underrepresented groups and disadvantaged groups, stating that: 'Higher education institutions will recruit, develop and retain high-quality staff, fully accountable for their performance to a strong and dynamic leadership' (DoES 2011, 27). Absent from the document is any reference to the under-representation of women in senior posts in higher education. Over the last five years, however, there have 
been major changes in Ireland within higher education in the promotion of gender equality. This is evident at sectoral, national and institutional policy levels. A number of developments have altered the national discourse. The first involved legal cases taken by a number of individual women academics in pursuit of promotional opportunities (Irish Human Rights and Equality Commission Report 2014). The second was the national adoption by the HEA, in 2015, of the Athena SWAN programme of awards to progress gender equality and as a means of stimulating change. Additionally, in 2013 the Irish Research Council (IRC), the main funding body for cross-disciplinary research, published its Gender Strategy and Action Plan 2013-2020. It focused on the streamlining and development of strategies to tackle gender imbalances across its programmes.

During this period a number of higher education institutions began to publish strategic plans that referenced gender, or published specific gender strategies (IADT 2019; TCD 2014; UCC 2017). The HEA Review of 2016 reinforced the political motivation to effect real change leading to a Gender Action Plan for Higher Education (HEA 2016). The establishment of a Government Equality Taskforce built upon the work of this review (HEA 2018). Among its recommendations for action was the announcement of a Senior Academic Leadership Initiative (SALI) to fund 45 women-only senior posts in higher education institutions. This initiative was introduced in response to statistics demonstrating that if the current pace of change in promoting women into senior posts continued, the imbalance would persist for another two to three decades. The initiative was also part of a commitment from the female Minister of State for Higher Education to tackle gender inequality in higher education institutions. The granting of the first 20 posts, 15 to universities, two for technological universities and three to institutes of technology, were announced in January 2020, following a competitive application process and assessment of the applications by an international panel of experts in the field of gender equality in higher education. The SALI initiative bolsters the stated aim of the government to have 40 per cent of chair professors posts held by women by 2024 (HEA 2019b).

The SALI initiative was not universally welcomed. Whilst some leaders and lecturing staff in higher education, both male and female, regarded it as a necessary step to tackle gender imbalances, others viewed it as tokenism and feared that it would not address the fundamental issues of culture, attitudes and equality. Some also expressed a concern that the women appointed to these posts would be ghettoised, regarded as second-class professors appointed on the basis of gender rather than academic merit. In 2019, the HEA set up a Centre of Excellence in Gender Equality to monitor its own progress around gender equality and progress in the sector. Its stated objective is: 'to ensure sustainable acceleration towards gender equality through centralised support for HEIs and dissemination of good practice' (HEA 2019c). The Centre is currently responsible for the SALI initiative, in place since the research for this chapter took place.

The key developments outlined previously refer directly or indirectly to the role of funding as a way of progressing gender equality. The actions were specifically 
targeted at women, ranging from actively encouraging them to apply for funding or posts, women-only programmes, and progress towards gender equality in the allocation of state funding for higher education institutions and research.

\section{The gender equality policy context}

The adoption of the Athena SWAN Charter in 2015 marked a turning point in the formalisation of progress towards addressing gender imbalance. Institutions that sign up to the Athena Swan Charter are required to carry out a self-assessment, analysing gender imbalances across a range of areas. These include an examination of gender-related policies, such as availability and take-up of flexible working arrangements, recruitment and promotion procedures and outcomes and staffing levels according to gender. This is accompanied by a Gender Action Plan, addressing any areas of concern arising from the self-assessment. The institution applies for an award based on its self-assessment and the robustness of its Gender Action Plan. It is also a requirement for institutions to hold an Athena SWAN award before individual departments or schools can apply for their awards. The HEA requires that all Irish HEIs must achieve an institutional Athena SWAN Bronze award to be eligible for funding by the end of 2020, with a requirement to have attained a Silver Award within seven years (HEA 2016, 291). Furthermore, progress around gender equality is required as a pre-requisite for research funding:

Within three years, research funding, as part of the Strategic Dialogue process, Higher Education Institutions will be at risk of funding being withheld if they are not addressing gender inequality sufficiently.

(HEA 2016, 91)

As a result of this report, a Gender Equality Taskforce was set up in 2017. It published a three-year action plan to identify good practice and highlight, as well as address, areas that needed improvement. The Gender Action Plan 2018-2020 states that: 'All HEIs shall submit their institutional gender action plan to the HEA and provide annual progress updates' (HEA 2018, 21). This led to a strategic dialogue whereby Irish HEIs must set and review targets annually with the HEA articulated via a Compact, a set of agreed targets and performance indicators, which include monitoring of the Gender Action Plan through a system of follow-up evaluation and performance monitoring, linked to funding. With funding linked to institutional performance, it could be withheld if institutions fail to meet the agreed KPIs and targets as set out in their Compact (HEA 2018).

\section{The research}

The research for this chapter was carried out between May 2018 and January 2020, gathering the views of leaders of the key funding bodies on the relationship between gender and funding. It was important to ascertain whether a threat 
to withhold funding for non-compliance could be a feasible and useful tool to promote gender equality. First, a desk-based review of the key policy documents was used to analyse specific commitments to the withholding of funding as a mechanism for influencing change towards achieving gender equality. Published frameworks provide the written declaration to use funding as a way of furthering gender equality. Second, a key question was how willing were funding bodies to implement the policy to affect meaningful change? To address this question, faceto-face semi-structured interviews were carried out with four leaders of funding bodies: the Department of Education and Skills (DoES); Irish Research Council (IRC); Higher Education Authority (HEA); and Health Research Board (HRB).

\section{Policy documents: a context for change?}

Overall, the political framework since 2015 has recognised the importance of funding as an incentive to address gender imbalances in higher education. One important dimension to affecting change is how the leaders of Irish HEI funding agencies see their role in implementing policies, and how effective they believe funding incentives to be. Key stakeholders were identified in four of the main funding bodies - the Department of Education and Skills, the Higher Education Authority, the Irish Research Council and the Health Research Board. At the time of the research interviews, all heads of these institutions were male. Before presenting the research and its findings, a short description of each of these five agencies is provided.

\section{Department of Education and Skills}

The Department of Education and Skills (DoES) is responsible for education and training. It produces an annual Action Plan for Education. The DoES (2019) plan includes reference, amongst its strategic priorities, to the need to address the gender-staffing imbalance in higher education. The DoES has a number of sections with specific responsibilities, including higher education. A Minister of State for Higher Education was appointed to lead a number of initiatives to address gender imbalance, including the SALI initiative.

\section{Higher Education Authority}

The Higher Education Authority (HEA) is the state body that leads on the strategic development of the Irish higher education and research system, with the objective of creating a coherent system of diverse institutions with distinct missions, responsive to the social, cultural and economic development of Ireland and its people, supporting the achievement of national objectives. The HEA has played a major role in commissioning and publishing strategic reports and policies on gender inequality. In 2019, it set up the Centre for Excellence in Gender Equality. The HEA is the statutory agency responsible for the allocation of exchequer 
funding to the universities, Institutes of Technology (IoTs) and other higher education institutions. The priorities of the HEA's Strategic Plan 2018-2022 include the implementation of the recommendations of the Report of the Expert Group, the HEA National Review of Gender Equality in Irish Higher Education Institutions and the Gender Equality Taskforce.

\section{Science Foundation Ireland}

Science Foundation Ireland (SFI) funds research in the areas of science, technology, engineering and mathematics. SFI seeks to remove and mitigate any existing or perceived factors that may limit the participation of women in research Sciences, Technology, Engineering and Mathematics (STEM) careers and to redress gender imbalances amongst SFI award holders, of whom 26 per cent were female (SFI 2018). One of SFI's key performance indicators (KPI) targets is to increase the proportion of female research award holders to 30 per cent by 2020 . This aim would facilitate the retention of excellent female researchers within academia, thereby increasing excellence in research and impact by continuing to fund meritorious researchers regardless of gender, through widening the pool of potential applicants.

\section{The Health Research Board}

The Health Research Board (HRB) is a state agency that funds and supports research and provides evidence to prevent illness, improve health and transform patient care. It published a HRB Policy on Gender in Research Funding (2019), outlining its intention to support both women and men to realise their full potential in order to ensure equality of opportunity and to maximise the quantity and the quality of research. The policy states that the HRB will work to achieve a greater gender balance throughout its research funding programmes and practices by: promoting gender equality within its own administration of research funding; and within the research teams that it funds; fostering the integration of sex and/ or gender into research and innovation content; and assuming a greater national responsibility for promoting gender equality in health research.

\section{Irish Research Council}

The Irish Research Council (IRC) is the body in Ireland that funds research across a range of disciplines. The IRC actively promotes gender equality in research funding and has set up a scheme to promote gender equality in research. It has published its own Gender Strategy 2013-2020 and is committed to reviewing and monitoring gender in research, including the numbers of women researchers and the amounts of funding they receive (IRC 2013).

These five funding bodies comprise the institutional framework for policymaking in relation to gender equality in higher education in Ireland. As such, they 
have considerable opportunity to shape future action and innovation in this area, making the interviews with four of their leaders particularly timely.

\section{The interviews}

The interviews were based around the following questions:

- How do funders see their role?

- Is the threat of withdrawal of funding a good incentive in achieving gender equality?

- Is the overall approach characterised as carrot, stick or both?

- What levers do funders have to make change happen?

- What happens if gender targets are not met?

- What are the likely challenges and resistances to implementing the funding approach?

All interviews were carried out face-to-face and were recorded and transcribed. The responses are outlined in the following sections.

\section{How funders see their role}

Interviewees were asked how they perceived their role in relation to funding and gender. All stated that they had a leadership and strategic role and outlined the importance of buying into and owning the problem and the solutions relating to gender. As one said:

'I think it's important to be seen as taking ownership of the issue but the question I would ask is the sector taking ownership, does the sector accept that it is a really significant issue [gender equality] that needs to be addressed, or is it a question of what is being done to them, being imposed?'

Another recognised that their organisation 'needed to take a leadership role in this'. The issue of leaders taking control themselves was a recurring theme:

'A key role for my organisation is an oversight role, an enabling role, but not doing the 'in the trenches' stuff that you are doing in the institutions'.

One leader pointed out that his organisation did not have a specific brief around funding individual institutions, stating that their focus was on the overall extent of sex and gender research content in applications for funding. He did state, however, that he was very conscious of his role as an influencer of gender equality.

All interviewees believed their role to be developers of policy and to ensure that initiatives were in place as part of their policy in relation to gender. All believed 
that they had a role in influencing the culture and that changing the organisational culture lay at the heart of ongoing strategic and structural transformation.

One leader said he recognised that these cultural differences might be granular, down to departmental level, as well as at institutional level:

'I think there are issues like different cultures within disciplines and departments in institutions where women have been in a minority. There are certain practices that have become part of the culture and disadvantaged women such as meetings in the evenings or early morning'.

One participant believed that changing the culture might take a long time, saying that:

'A lot of the structures, but above all the culture that is in place, is the product of a long time and is not going to be undone with a five year plan'.

Another also highlighted the role of culture:

'We need to ask the question about the culture of higher education and whether male dominated roles are perpetrating what you hope are legacy bad practices, concerned that the culture of higher education isn't what it should be on account of male dominated practice and a nationwide lack of gender balance'.

The belief that changing culture and attitudes lies at the heart of ongoing strategic and structural transformation was evident in all responses. All four interviewees spoke about their role as influencers, but expressed a view that until institutional leaders identified and rewarded good practice, no real change would be effective. All interviewees reiterated that their organisations had a monitoring and review responsibility to ensure that the recommendations and required actions, identified in key policy documents, were implemented.

\section{A carrot or stick approach?}

Leaders of the HEA and the DoES acknowledged that ultimately they had the power to impose funding sanctions for the higher education institutions that do not make progress in gender equality. These two interviewees, who identified their potential to utilise the withholding of funding as a sanction, were asked what specific incentives (carrots) or sanctions (sticks) they had at their disposal. The DoES and HEA identified specific monetary sanctions and the imposition of additional formal monitoring for those HEIs deemed not to be performing well. They also referred to progress on Gender Action Plans by HEIs.

All the interviewees were supportive of the Athena SWAN accreditation requirement and the withholding of funding, with one saying that:

'Ultimately it is about outcomes but if you want to play evaluation in a system you have to be able to tell that system that this is how the outcome will 
be judged. That is why we ended up having an evaluation mechanism like Athena SWAN'.

Another pointed out:

'In terms of realism, it's hard to see how gender could be addressed in the timeframes set but if no timeframes are set no progress will be made so Athena Swan accreditation is an important first step'.

All of the interviewees believed that there is also a need for positive measures, funding rewards as well as imposing sanctions. One stated that if the whole of academia had to be 'dragged kicking and screaming' to realise gender objectives, on account of the risk that they might lose funding, then real change and commitment might be limited. He did accept, however, that there had to be consequences for those not making progress and that one clear sanction was monetary loss. He went on to say that he recognised that this approach came with challenges. When pressed on whether the approach of the four institutions was mainly carrot or stick, all interviewees identified the need for a mixture of both approaches. The overall response was that they preferred the carrot approach alongside institutional ownership of the gender equality issue at a local level. However, they all stated that they would use the stick approach as necessary.

\section{What funding levers are available?}

Interviewees were asked about their ability to use the stick as a lever, in terms of whether it was feasible and possible to withhold funding. One pointed out that one way this could happen was through use of Compact, where targets and actions are reviewed annually by the HEA and an international panel. At this point funding could be withheld from HEIs. Another referenced performance review, pointing out that:

'Performance dialogue is important, in that there can be a number of priorities including gender in these discussions. The role of the Department [DoES] is in saying we want that issue to be front and centre and there to be real reasons for people who clearly are not compliant or who do not understand the need for change'.

He also pointed out that there had to be real consequences for inaction, given the government's stated commitment to gender equality. All interviewees approved the approach advocated by the Report of the Gender Task Force Expert Group (HEA 2018) relating to the withholding of funding. An example given was institutions not achieving Athena SWAN accreditation within the required time. One interviewee stated:

'The funding leverage is very powerful and can help to progress things that might not happen so quickly otherwise'. 
Interviewees were also asked if they supported positive action initiatives, specifically funding incentives or reward for good progress. One leader pointed out that his organisation already had a number of positive actions as part of its gender strategy and action plan. These included campaigns to highlight the achievements of women researchers, gender-blind research assessments and a requirement for research proposal assessment panels to be comprised of at least 40 per cent women. Career breaks, due to looking after children and other family members were included when assessing an applicant's record of accomplishment. Another interviewee believed that:

'Lobbying for any uplift in research funding has to be cross departmental and cross agency so that the Irish Research Council can expand their programmes as well as Science Foundation Ireland and the Health Research Board'.

They all supported the use of funding as an incentive and stated that many of their organisational processes already implemented this. Most were committed to consistently reviewing their funding practices to ensure fairness and equity. Statistics on the awarding of funding, by gender, are also gathered and reviewed in all organisations. One interviewee stated his support for a positive funding approach, positing the idea of:

'rewarding or incentivising those institutions that have shown that they can embrace this agenda, that they are changed and forging ahead'.

Another participant pointed out that, at the time of the interview, the Gender Task Force was looking at rewarding those bodies that had made good progress. He supported this and believed it would be helpful if there were some measures to reward good performance, alongside the sanctions.

Interviewees were asked about quotas and sanctions for not meeting targets, for example, the withholding of funding if an institution did not reach 50:50 senior academic posts within an agreed timeframe. The Report of the Gender Task Force Expert Group (HEA 2016) had referenced quotas for academic promotions, based on a flexible cascade model where the proportion of women and men to be promoted or recruited is based on the proportion of each gender at the grade immediately below. The HEA refers to: 'A minimum of 40 per cent women and 40 per cent men to be full professors at the appropriate pay scales' to be achieved by the end of 2024. One leader pointed out that his organisation had set targets for female membership of assessment panels and individual scholarships for researchers, but it had not used quotas in the awarding of funding. He did state that he wanted:

'very much to try and work with the stakeholders such as the HEA, to ensure that the IRC makes a positive contribution to the landscape in this area'.

Another interviewee was interested in the complexities of the system and whether the introduction of quotas would be effective. He pointed out that there had been 
considerable discussion in the Gender Task Force Expert Group's work on that issue (HEA 2018). In particular, using a cascade model of appointing women to senior posts where they became available, would still take two decades for gender equality to be reached. He did point out that there were actions that were not quotas that could be considered:

'There have been examples in other EU foundations, other states, where they have pushed the envelope on the civil service into level roles, for example if two candidates are equivalent, preference can be given to the female candidate under gender equality objectives'.

This begs the question as to why this would not be feasible in Irish academic institutions. Quotas did not appear to be something that any of the four institutional leaders were keen to introduce. Instead, rewards for good practice, which are only minimally in place at present, via funding from the HEA, could be developed. The current performance review system does allow for the withholding of funding but the new system was only partly in place in 2019. None of the interviewees reported any withholding of funding to institutions, to date.

All interviewees restated that without the situation where gender balance was supported, encouraged and led from the top, real change could not happen. They favoured buy-in and a cultural shift as the real and sustainable agent of change. They all had concerns about the length of time it would take to effect change. All four interviewees expressed the view that a robust system of sanctions and rewards, based on real evidence, was needed alongside every opportunity and support being given to individual institutions to change.

\section{Challenges and resistance}

Interviewees were asked to outline any challenges that they might face in implementing national policy towards gender equality. More specifically, they were asked to identify structural challenges to implementing change, particularly around contractual issues preventing institutions from meeting their targets. For example, in seeking to increase female representation in senior posts, or institutions not being able to implement the flexible cascade model, would funding be withheld?

All interviewees confirmed that some current practices might be difficult to change quickly, but all felt that this was an area that could be achieved in partnership with the key stakeholders. They were also concerned that external factors, such as changes in political leadership or personnel in key roles, could pose challenges and might lead to slippage on the gender issue. All pointed out that progress made has been under the strong leadership of the current female Minister of State for Higher Education. As one interviewee stated:

'It could slip back, the main progress that has been made to now has been at the level of attention it gets'. 
He pointed out that unless gender actions are enshrined in policy and monitored then the focus on gender may change in the future saying: 'The challenge is to keep it there as a high priority'.

All leaders noted that the major issues were inaction, apathy and even hostility from some institutions and their leaders. One said:

'I heard one president saying [that] many institutions had to be dragged kicking and screaming into this'.

Getting institutional leaders firmly 'on board' with the equality agenda was defined by one interviewee as producing an action plan and taking real actions and ownership of the issue to initiate change. So the overall message here is that before cultural and attitudinal change happens, behavioural change, led by public body initiatives, must pave the way.

Academia in Ireland has faced a number of funding challenges over the last 20 years. Interviewees were asked if they had any other comments to make on funding and gender equality. One interviewee summed up the main challenge:

It is well recognised that we are right down the league tables in terms of funding of higher education and my question is - what is the impact of that on gender equality?'

\section{Conclusions}

The current policy frameworks for using funding as a sanction-led initiative in the battle for gender equality have been in place since Athena Swan was instigated in 2015. Subsequent reports and policy documents have outlined the expectation placed on higher education to improve its performance in relation to gender equality, particularly in women's representation in research and senior academic posts. Annual performance reviews are, in theory, linked to funding. Mechanisms exist for the withholding of funding where satisfactory progress against targets has not been met. Some positive action initiatives, including SALI, have been introduced in the last year, recognising that without these innovations, gender parity in senior posts will take decades to achieve.

Leaders of the four funding organisations all had a well-founded awareness of, and individual commitment to, gender equality. Their stated preference was for the changing of cultures, the winning of hearts and minds rather than the threat of withholding funding and telling institutions what they must do. Although the use of quotas was not the preferred option, all leaders favoured positive action initiatives, or had already introduced them. The approach in general was that good leadership and commitment from the funding bodies they represented had to be matched by strong leadership in the institutions themselves. All interviewees believe that there is an opportunity to utilise funding as both a sanction and a reward, although no evidence was presented of this happening to date. All 
recognised that failure to do so would mean another two decades of no real progress and continued dependence on the goodwill and commitment of individual leaders. In 2020, when the first round of HEA performance reviews will take place in Ireland, assessment of progress by individual HEIs, with their Gender Action Plans and progress towards the achievement of Athena SWAN, will be part of the assessment criteria. Using funding as both a sanction and a reward, with support from the CEOs of the State funding bodies, will be critical to this process.

\section{References}

DoES (2011) National strategy for higher education to 2030, Report of the Strategy Group, Department of Education and Skills, Government Publications Office, Dublin.

DoES (2019) Empowering through learning: Action plan for education 2019, Department of Education and Skills, Dublin.

HEA Expert Group (2016) HEA national review of gender equality in Irish higher education institutions, Higher Education Authority, Dublin.

HEA Expert Group (2018) Gender action plan 2018-2020: Accelerating gender equality in Irish higher education institutions, Higher Education Authority, Dublin.

HEA Expert Group (2019a) Higher education institutional staff profiles by gender 2019, Higher Education Authority, Dublin. Available at: https://hea.ie/assets/uploads/2019/07/ Higher-Education-Institutional-Staff-Profiles-by-Gender-2019.pdf

HEA Expert Group (2019b) Senior academic leadership Initiative (SALI) Call for applications2019 Promoting gender balance at senior academic levels in the higher education sector. Available at: https:/hea.ie/assets/uploads/2019/06/FINAL-Call-document-2019-06-21.pdf

HEA Expert Group (2019c) Centre of excellence for gender equality, HEA, Dublin. Available at: https://hea.ie/policy/gender/gender-equality-centre-for-excellence/

HRB (2019) HRB policy on gender in research funding, Health Research Board, Dublin. Available at: www.hrb.ie/funding/funding-schemes/before-you-apply/all-grant-policies/ hrb-policy-on-gender-in-research-funding/

IADT (2019) Strategic plan 2019-2023. Available at: www.iadt.ie/content/files/IADT_ Strategic_Plan_WEB_AW.pdf

Irish Research Council (2013) Gender strategy and action plan 2013-20, Irish Research Council, Dublin. Available at: http://research.ie/resources/publications/gender-strategyand-action-plan-2013-2020/

Irish Human Rights and Equality Commission Report (2014). Available at: www. workplacerelations.ie/en/cases/2014/November/DEC-E2014-078.html

SFI (2018) Annual report and accounts, Science Foundation, Ireland. Available at: www. sfi.ie/research-news/publications/annual-reports/sfi-annual-report-2018/

TCD (2014) Strategic plan 2009-2014. Available at: www.tcd.ie/strategy/strategic-plan201419.pdf

UCC (2017) Strategic plan 2017-2022. Available at: www.ucd.ie/president/t4media/UCDStrategy2015-2020.pdf 